Article

\title{
Evaluation of Organ Dose and Image Quality Metrics of Pediatric CT Chest-Abdomen-Pelvis (CAP) Examination: An Anthropomorphic Phantom Study
}

\author{
Nor Azura Muhammad ${ }^{1}$, Zunaide Kayun ${ }^{1}$, Hasyma Abu Hassan ${ }^{2}$, Jeannie Hsiu Ding Wong ${ }^{3}$, \\ Kwan Hoong $\mathrm{Ng}^{3}$ and Muhammad Khalis Abdul Karim ${ }^{1, * \mathbb{D}}$ \\ 1 Department of Physics, Faculty of Science, University Putra Malaysia, Serdang 43400, Malaysia; \\ norazura11@gmail.com (N.A.M.); zunaide@moh.gov.my (Z.K.) \\ 2 Department of Radiology, Faculty of Medicine, University Putra Malaysia, Serdang 43400, Malaysia; \\ hasyma@upm.edu.my \\ 3 Department of Biomedical Imaging, University Malaya Medical Centre, \\ Petaling Jaya 59100, Kuala Lumpur, Malaysia; jeannie.wong@ummc.edu.my (J.H.D.W.); \\ ngkh@ummc.edu.my (K.H.N.) \\ * Correspondence: mkhalis@upm.edu.my
}

check for updates

Citation: Muhammad, N.A.; Kayun, Z.; Abu Hassan, H.; Wong, J.H.D.;

$\mathrm{Ng}$, K.H.; Karim, M.K.A. Evaluation of Organ Dose and Image Quality Metrics of Pediatric CT ChestAbdomen-Pelvis (CAP) Examination: An Anthropomorphic Phantom Study. Appl. Sci. 2021, 11, 2047. https:// doi.org/10.3390/app11052047

Academic Editor: Salvatore Gallo

Received: 25 January 2021

Accepted: 11 February 2021

Published: 25 February 2021

Publisher's Note: MDPI stays neutral with regard to jurisdictional claims in published maps and institutional affiliations.

Copyright: (c) 2021 by the authors. Licensee MDPI, Basel, Switzerland. This article is an open access article distributed under the terms and conditions of the Creative Commons Attribution (CC BY) license (https:// creativecommons.org/licenses/by/ $4.0 /)$.
Featured Application: We evaluate and propose a method for evaluating optimized CT protocol for pediatric-based figure of merit and image performance metrics.

Abstract: The aim of this study is to investigate the impact of CT acquisition parameter setting on organ dose and its influence on image quality metrics in pediatric phantom during CT examination. The study was performed on 64-slice multidetector CT scanner (MDCT) Siemens Definition AS (Siemens Sector Healthcare, Forchheim, Germany) using various CT CAP protocols (P1-P9). Tube potential for P1, P2, and P3 protocols were fixed at $100 \mathrm{kVp}$ while P4, P5, and P6 were fixed at $80 \mathrm{kVp}$ with used of various reference noise values. P7, P8, and P9 were the modification of P1 with changes on slice collimation, pitch factor, and tube current modulation (TCM), respectively. TLD-100 chips were inserted into the phantom slab number 7, 9, 10, 12, 13, and 14 to represent thyroid, lung, liver, stomach, gonads, and skin, respectively. The image quality metrics, signal to noise ratio (SNR) and contrast to noise ratio (CNR) values were obtained from the CT console. As a result, this study indicates a potential reduction in the absorbed dose up to $20 \%$ to $50 \%$ along with reducing tube voltage, tube current, and increasing the slice collimation. There is no significant difference $(p>0.05)$ observed between the protocols and image metrics.

Keywords: computed tomography; absorbed dose; signal-noise ratio; contrast-noise ratio; figure of merit

\section{Introduction}

The transformation and sophistication of the computed tomography (CT) system have led to the increased use of CT examinations. The development of CT technology begins from the single detector and now the multidetector CT (MDCT) able to produce high contrast sectional images in short scanning time [1,2]. Recent advances in the technological breakthrough of CT scan performance, such as application of automatic tube current modulation (ATCM) and utilization of iterative reconstruction algorithm technique, have decreased CT radiation exposure while retaining diagnostic image consistency [3-5].

The imaging of pediatric CT examination is still challenging even though the performance and technology of the latest generation of CT scanners have been introduced. Recently, with growing involvement in radiation-induced cancer, this debate is again in the spotlight, as radiation exposure optimization is a particular problem in pediatric patients receiving repeated CT examination [6-8]. Despite the high frequency of CT procedure 
usage amongst children, radiation burden from CT examination needs to be considered as children are more sensitive to radiation as compared to adults [9,10]. Since 2007, the American College of Radiology (ACR), the Society for Pediatric Radiology (SPR), the American Association of Physicists in Medicine (AAPM), and the American Society of Radiology Technologists (ASRT) have engaged in the Image Gently campaign in the United States to grow the knowledge of practice by increasing awareness among radiation personnel [11,12].

Generally, the radiation-absorbed dose can be measured by inserting a passive dosimeter inside anthropomorphic phantom as it is the accurate way to measure the radiation absorbed by radiosensitive organs or tissues of patients [13-15]. Likewise, Monte Carlo estimation on computational phantom, such as CT-Expo (Version 2.3.1, Hannover, Germany), is able to estimate an organ-absorbed dose and effective dose based on the subject's age and gender [16-18]. In addition, multiplying organ-absorbed dose with tissue weighting factor as stated in the International Commission on Radiological. Protection (ICRP 103) can also be used to calculate the value of effective dose which is an important radiation metric for the estimation of cancer risk of individual patients $[19,20]$.

CT chest-abdominal pelvis (CAP) examination is generally used to evaluate the anatomy structures and to diagnose the degree of malignancies in lungs, liver, pelvic organ, lymphatic nodules, and bony structures. According to Park et al. [21], CT CAP examination was performed purposely for the cancer staging. The mean effective dose of pediatric CT CAP examination was ranging from 3.4 to $7.5 \mathrm{mSv}$ between one year and five years of age [22,23]. Hence, pediatric patients who require CT CAP scans due to their health problems may have an elevated chance of cancer because children are more vulnerable to radiation effects than adults [24]. CT dose reduction protocols have been developed amongst researchers and a variety of strategies have been established to minimize CT dose without compromising diagnostic image quality [25-27]. These include reducing tube current (mAs) and tube voltage $(\mathrm{kVp})$, automatic attenuation-based tube current selection, decreased pitch factor, increased slice collimation and improper positioning [28,29]. Several researchers have proposed CT dose reduction by increasing the pitch value combined with the iterative reconstruction method to obtain significant dose reduction of the normal chest and abdominal scans while preserving the consistency of the image quality [28,30,31].

The vigorous action by the institution in setting the best optimization protocols, particularly for pediatric patients, is an indispensable part in reducing dose exposure in CT examination. Therefore, the study highlights the current finding in optimizing radiation doses from altering pediatric CT CAP scan parameters in terms of tube voltage, tube current, slice collimation, pitch, and effect of tube current modulation (TCM) application, as well as the effect on image quality indices in regard of image noise, signal to noise ratio (SNR), and contrast to noise ratio (CNR).

\section{Materials and Methods}

This study utilized $1 \mathrm{y} / \mathrm{o}$ anthropomorphic phantom (ATOM, 704 model CIRS, Norfolk, VA, USA). The body weight, height and thorax dimension of the phantom were $10 \mathrm{~kg}$, $75 \mathrm{~cm}$, and $12 \times 14 \mathrm{~cm}$, respectively. This phantom was made from tissue equivalent material, which consisted of the lung, soft, and bone tissue. The phantom was made up of 28 slabs with $2.5 \mathrm{~cm}$ slice thickness, which cover from head to lower leg and each slab contains a hole about $5.0 \mathrm{~mm}$ in diameter for inserting dosimeter, such as TLD, to measure the absorbed dose at the specific area of sensitive organs and for the simulation study of a blood vessel filling with contrast material, as shown in Figure 1. 


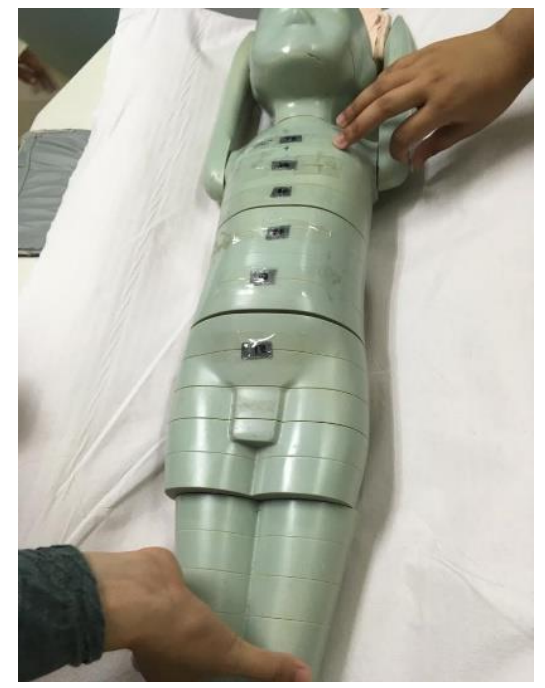

(a)

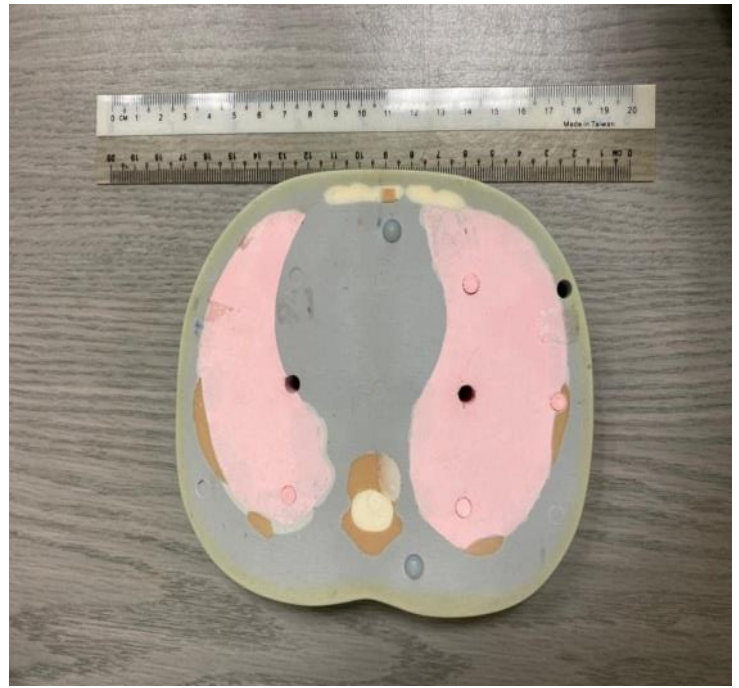

(b)

Figure 1. (a) One-year-old anthropomorphic phantom was set up before the scan. (b) The anthropomorphic lung tissue slab comprises a-holes matrix about $5.0 \mathrm{~mm}$ in diameter for dosimeter insertion, such as TLD.

The CT CAP examination was performed on 64 slices using a multidetector CT scanner (MDCT) Siemens Definition AS (Siemens Sector Healthcare, Forchheim, Germany) by applying nine different $\mathrm{CT}$ protocols ( $\mathrm{P} 1$ to $\mathrm{P} 9$ ). The tube potential of $\mathrm{P} 1, \mathrm{P} 2$, and $\mathrm{P} 3$ were fixed at $100 \mathrm{kVp}$ and $\mathrm{P} 4, \mathrm{P} 5$, and $\mathrm{P} 6$ were fixed at $80 \mathrm{kVp}$ with various tube current reference values (ref. mAs: 210, 180, and 150), while in P7, P8, and P9 the modification was made on P1 with changes on slice collimation, pitch factor, and tube current modulation (TCM), respectively. The details of the CT acquisition parameter used in this study were shown in Table 1 . The scan range starts from $1 \mathrm{~cm}$ above the apex of lungs and ends at $1 \mathrm{~cm}$ below symphysis pubis with a total length of $32 \mathrm{~cm}$ for all protocols involved. The source to detector (SDD) and source to object distance (SOD) used in this study were $100 \mathrm{~cm}$ and $50 \mathrm{~cm}$, respectively.

Table 1. Routine and modified CT CAP acquisition parameters used in this study.

\begin{tabular}{|c|c|c|c|c|c|c|c|c|c|}
\hline \multirow{2}{*}{ Parameter } & \multicolumn{9}{|c|}{ Protocols } \\
\hline & P1 & $\mathbf{P 2}$ & P3 & $\mathbf{P 4}$ & P5 & P6 & P7 & P8 & P9 \\
\hline Slice thickness recon (mm) & 3 & 3 & 3 & 3 & 3 & 3 & 3 & 3 & 3 \\
\hline Tube potential $(\mathrm{kVp})$ & 100 & 100 & 100 & 80 & 80 & 80 & 100 & 100 & 100 \\
\hline Effective tube current (mAs) & 27 & 23 & 19 & 33 & 31 & 26 & 27 & 26 & - \\
\hline Ref. mAs & 210 & 180 & 150 & 210 & 180 & 150 & 210 & 210 & 210 \\
\hline Number of detector & 64 & 64 & 64 & 64 & 64 & 64 & 32 & 64 & 64 \\
\hline Slice collimation (mm) & 0.6 & 0.6 & 0.6 & 0.6 & 0.6 & 0.6 & 1.2 & 0.6 & 0.6 \\
\hline Pitch & 1.4 & 1.4 & 1.4 & 1.4 & 1.4 & 1.4 & 1.4 & 1.2 & 1.4 \\
\hline Dose Modulation & On & On & On & On & On & On & On & On & Off \\
\hline Table Feed & 53.8 & 53.8 & 53.8 & 53.8 & 53.8 & 53.8 & 53.8 & 56.0 & 53.8 \\
\hline Exposure time (s) & 0.5 & 0.5 & 0.5 & 0.5 & 0.5 & 0.5 & 0.5 & 0.5 & 0.5 \\
\hline Kernel filtration & B30f & B30f & B30f & B30f & B30f & B30f & B30f & B30f & B30f \\
\hline $\mathrm{CTDI}_{\mathrm{vol}}(\mathrm{mGy})$ & 1.8 & 1.5 & 1.3 & 1.0 & 0.9 & 0.8 & 1.7 & 1.7 & 6.9 \\
\hline DLP (mGy.cm) & 58 & 48 & 42 & 32 & 29 & 26 & 54 & 54 & 221 \\
\hline $\mathrm{E}$ (DLP $\times k$ factor) & 1.6 & 1.3 & 1.2 & 0.9 & 0.8 & 0.7 & 1.5 & 1.5 & 6.2 \\
\hline
\end{tabular}




\subsection{CT Radiation Dose}

Dose data, such as volume weighted CT Dose Index $\left(\mathrm{CTDI}_{\mathrm{vol}}\right)$ and dose length product (DLP), were collected from the CT console. $\mathrm{CTDI}_{\mathrm{vol}}$ is the most established radiation metric for $\mathrm{CT}$ and considered an important value for quality assurance. $\mathrm{CTDI}_{\mathrm{vol}}$ is defined as:

$$
\mathrm{CTDI}_{\mathrm{vol}}=\frac{\mathrm{CTDI}_{\mathrm{w}}}{\text { pitch factor }}
$$

where $\mathrm{CTDI}_{\mathrm{W}}$ is the weighted average dose across a single slice and pitch is the distance of the table moving in one $360^{\circ}$ gantry rotation. DLP is calculated by multiplying the scan range of the patients with the $\mathrm{CTDI}_{\mathrm{vol}}$, and it represents the overall dose output along the z-axis. Furthermore, the effective dose (E) is estimated by multiplying the DLP with a conversion coefficient factor (CC) [13].

The organ dose of the phantom was measured by using TLD-100 (LiF: Mg, Ti) with dimensions of $3.2 \times 3.2 \times 0.6 \mathrm{~mm}$. TLD-100 is the common passive dosimeter used in the measurement of radiation dose due to high sensitivity and response to the photon of X-ray. A total of 55 TLD-100 were used in this study and calibrated and compared with standard ionization chamber. The calibration process should be done to obtain a conversion factor (CF) in each TLD-100. Nine TLD-100 were used to measure background radiation. The CF was computed based on the equation:

$$
\mathrm{CF}=\frac{\mathrm{Q}_{\text {rad }}}{\left(\mathrm{M}-\mathrm{M}_{\text {background }}-\mathrm{C}_{\text {background }}\right)}
$$

where $Q_{\text {rad }}$ is energy photons from X-ray beam, $M$ is the result of the Harshaw TLD reader, $\mathrm{M}_{\text {background }}$ is the background reading from TLD reader, and $\mathrm{C}_{\text {background }}$ is the background reading from TLD-100.

All the TLD-100 were read out using a Harshaw 3500 TLD reader (Thermo Scientific, Waltham, MA, USA), combined with an operating software for all Harshaw TLD reader known as WinREMS after 24-h exposure. Before reuse, the TLD-100 chips were annealed using an oven (TLD annealing oven, PTW, Freiburg, Germany) for $1 \mathrm{~h}$ at $400{ }^{\circ} \mathrm{C}$ and $2 \mathrm{~h}$ at $100{ }^{\circ} \mathrm{C}$ followed by rapid cooling to make the TLD chips reusable. All the TLD-100 kept inside labeled plastic and sealed before inserting into the hole of the phantom. In this study, four TLD chips were inserted into the phantom slab no 9, 12, and 14 to represent lung, liver, and stomach, three TLD were inserted into slab no 7 and 19 to represent thyroid and gonads, and five TLD was placed on top of the phantom at the area of the thorax (slab number 8 and 11), abdomen (slab number 12 and 13), and pelvis (slab number 18) for the skin dose measurement.

\subsection{Image Quality Metrics}

The image quality was assessed by using Radiant DICOM Viewer software (Medixant, Poznan, Poland) to extract CT numbers and noise values from each protocol by placing the circular region of interest (ROI) about $0.8 \mathrm{~cm}^{2}$ in the selected image (Figure 2). The information regarding CT numbers and noise was used to calculate the signal to noise ratio (SNR) and contrast to noise ratio (CNR). The SNR was calculated by dividing the mean (CT number) by the corresponding standard deviation, $\mathrm{SD}$ (noise value) as shown in the equation below:

$$
\mathrm{SNR}=\frac{\text { mean }_{\mathrm{ROI}}}{\mathrm{SD}_{\mathrm{ROI}}}
$$

Furthermore, the CNR were measured by using the equation below:

$$
\mathrm{NR}=\frac{\mid \text { mean }_{\mathrm{ROI}}-\text { mean }_{\mathrm{BG}} \mid}{\frac{\mathrm{SD}_{\mathrm{ROI}}+\mathrm{SD}_{\mathrm{BG}}}{2}}
$$


where the mean BG $_{\mathrm{B}}$ and $\mathrm{SD}_{\mathrm{BG}}$ is $\mathrm{CT}$ number value of background and noise value of the background, respectively. We quantify the dose information to correlate with the CT image quality. The figure-of-merit (FOM) was used to characterize the radiation dose trade-off with image quality by considering all the image parameters. In this study, the FOM values represent the efficiency between SNR and $\mathrm{CTDI}_{\mathrm{vol}}$ with different CT CAP protocols and they can be calculated with the equation below:

$$
\mathrm{FOM}=\frac{\mathrm{SNR}^{2}}{\mathrm{CTDI}_{\mathrm{vol}}}
$$

Besides SNR, CNR also can be used to calculate the FOM with the CTDI ${ }_{\text {vol }}$ parameter. The FOM allows SNR to be evaluated independently of the current tube and the dose of radiation. However, as it is determined for a specific scan mode (helical or axial) and a specific diagnostic task, it should not be used, for example, when comparing head and abdomen imaging, to compare different scanned areas of diagnostic tasks [22].

Data were analyzed using IBM SPSS V25.0 (SPSS, version 25.0 for Windows, Chicago, IL, USA). The data of organ-absorbed dose were presented as mean value with standard deviation (SD). One-way ANOVA with post-hoc Dunnett test was used to analyze the significant value of organ-absorbed dose and image quality between default protocol 1 (P1) with other modified protocols CT CAP. A $p$-value $<0.05$ was considered statistically different.

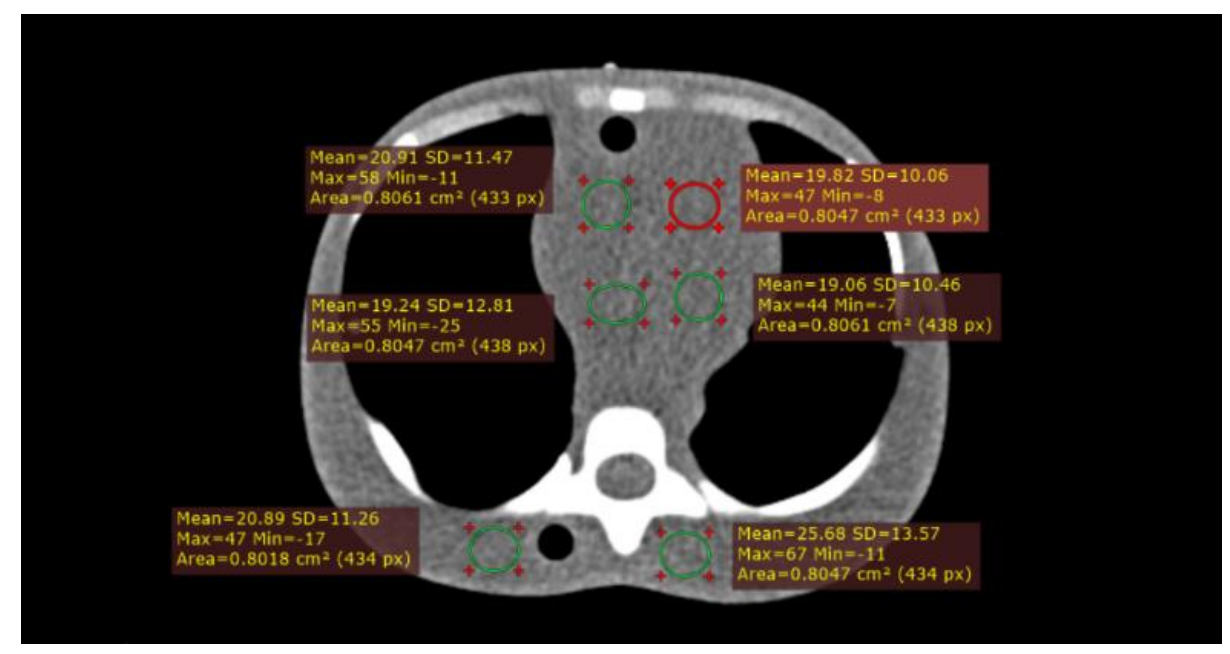

Figure 2. Example of objective image quality analysis of CT number (mean), image noise (SD), SNR, and CNR by putting the region of interest (ROI) in the lungs and soft tissue field.

\section{Results}

\subsection{Organ Equivalent Dose}

Table 2 summarized the organ equivalent dose for the selected organs in protocols P1-P6. As observed, the highest organ-absorbed dose was found in the liver and the lowest was presented in the thyroid for $100 \mathrm{kVp}$ with a mean value of $1.22 \pm 0.13 \mathrm{mSv}$ (P1) and $0.31 \pm 0.07 \mathrm{mSv}$ (P3), respectively. However, for $80 \mathrm{kVp}$, the highest organabsorbed dose was found in the stomach compared to the other organs with a mean value of $0.72 \pm 0.01 \mathrm{mSv}$. Figure 3 presents the mean organ equivalent doses of standard pediatric CT CAP examinations with varying tube potential and tube current. As a result, the value of doses reduced as tube voltage and tube current decrease in P2 to P6. In comparison between 100 and $80 \mathrm{kVp}$, the results of organ-absorbed dose were decreased with the reduction of tube voltage. Overall, this study found that the organ dose measurements in thyroid, lungs, liver, stomach, gonads, and skin were reduced by almost $50 \%$ with lessening tube voltage from 100 to $80 \mathrm{kVp}$. 
Table 2. Organ Equivalent Dose from TLD measurement between 100 and $80 \mathrm{kVp}$ with variation ref. mAs.

\begin{tabular}{|c|c|c|c|c|c|c|}
\hline \multirow{3}{*}{ Selected Organ } & \multicolumn{6}{|c|}{ Organ Equivalent Dose (mSv) } \\
\hline & \multicolumn{3}{|c|}{$100 \mathrm{kVp}$} & \multicolumn{3}{|c|}{$80 \mathrm{kVp}$} \\
\hline & $\begin{array}{c}\text { P1 } \\
\text { (Ref. mAs 210) }\end{array}$ & $\begin{array}{c}\text { P2 } \\
\text { (Ref. mAs 180) }\end{array}$ & $\begin{array}{c}\text { P3 } \\
\text { (Ref. mAs 150) }\end{array}$ & $\begin{array}{c}\text { P4 } \\
\text { (Ref. mAs 210) }\end{array}$ & $\begin{array}{c}\text { P5 } \\
\text { (Ref. mAs 180) }\end{array}$ & $\begin{array}{c}\text { P6 } \\
\text { (Ref. mAs 150) }\end{array}$ \\
\hline Thyroid & $0.49 \pm 0.03$ & $0.41 \pm 0.05$ & $0.31 \pm 0.07$ & $0.24 \pm 0.16$ & $0.22 \pm 0.16$ & $0.20 \pm 0.17$ \\
\hline Lungs & $0.88 \pm 0.12$ & $0.74 \pm 0.06$ & $0.58 \pm 0.06$ & $0.51 \pm 0.04$ & $0.37 \pm 0.09$ & $0.35 \pm 0.05$ \\
\hline Liver & $1.22 \pm 0.13$ & $0.95 \pm 0.06$ & $0.83 \pm 0.06$ & $0.53 \pm 0.15$ & $0.46 \pm 0.12$ & $0.39 \pm 0.08$ \\
\hline Stomach & $1.05 \pm 0.03$ & $0.95 \pm 0.09$ & $0.82 \pm 0.07$ & $0.72 \pm 0.01$ & $0.62 \pm 0.04$ & $0.56 \pm 0.04$ \\
\hline Gonads & $0.87 \pm 0.02$ & $0.75 \pm 0.03$ & $0.64 \pm 0.06$ & $0.43 \pm 0.02$ & $0.40 \pm 0.01$ & $0.34 \pm 0.02$ \\
\hline Skin & $1.07 \pm 0.14$ & $0.90 \pm 0.16$ & $0.68 \pm 0.24$ & $0.59 \pm 0.14$ & $0.48 \pm 0.17$ & $0.32 \pm 0.16$ \\
\hline
\end{tabular}

Table 2 and Figure 3 present the impact of standard protocol, tube voltage, and tube current on organ equivalent dose during the examination. Table 3 summarizes the influence of increased slice collimation (P7), reduced pitch factor (P8), and disabling the TCM function (P9) on organ dose. The doses were slightly reduced when slice collimation was increased. However, by reducing the pitch value, the organ dose was slightly increased. Furthermore, the organ equivalent dose was increased by more than $50 \%$ in P9 after disabling the TCM function. Figure 4 shows the highest organ dose was observed in the liver followed by skin, stomach, lungs, gonads, and thyroid in all protocols. The highest organ dose was detected in P9. Therefore, the application of TCM in pediatric patients offers a big consequence in $\mathrm{CT}$ radiation dose due to different in-patient body habitus and thickness.

\subsection{Analysis of Image Quality Metrics}

The image quality metrics were obtained to compare with the dose information. The mean of image quality indices of CT number, noise, SNR, and CNR were summarized in Table 4 . The reduced tube voltage and tube current lead to an increase in image noise. Noting that the highest mean of noise was $13.1 \pm 0.6 \mathrm{HU}$ presented in P6. There is no significant difference of CNR in P4 and P6 after reducing the tube voltage from 100 to $80 \mathrm{kVp}$. However, reducing tube voltage in P4, P5, and P6 resulting in significant differences $(p<0.05)$ of SNR value. The highest mean of SNR and CNR in P9 (disabling TCM) was $6.66 \pm 0.5 \mathrm{HU}$ and $1.34 \pm 0.2 \mathrm{HU}$, respectively, and the organ dose in $\mathrm{P} 9$ protocol were among the highest. Thus, the highest FOM value was obtained in P9, followed by P7 and P2.

(a)

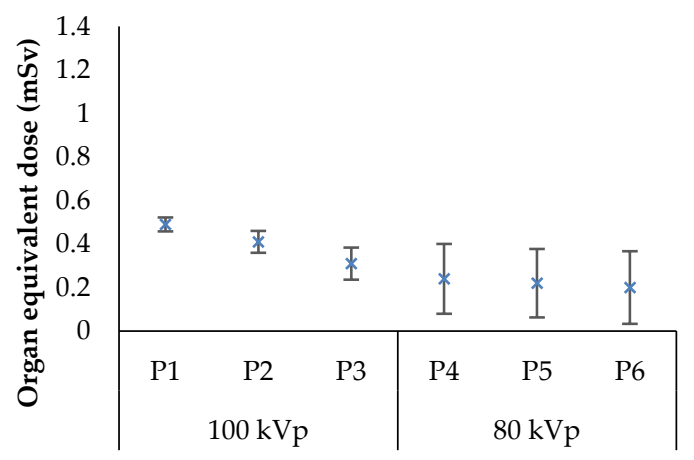

CT CAP Protocols (b)

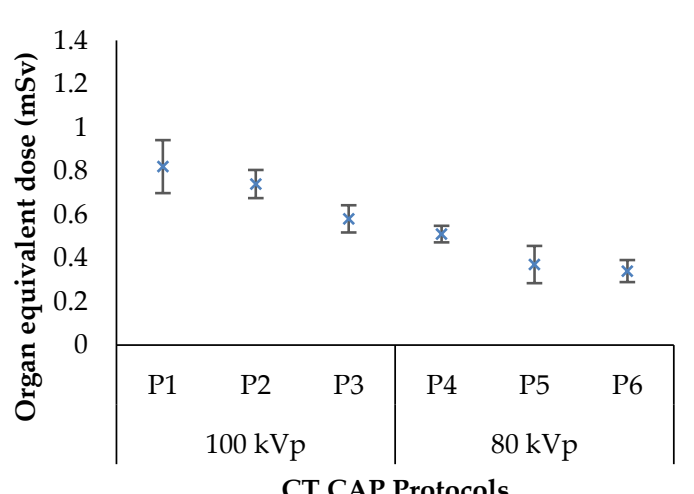

Figure 3. Cont. 
(c)

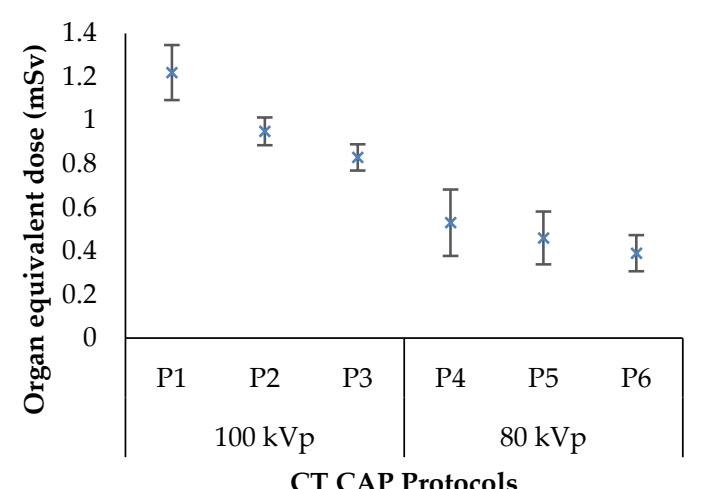

(e)

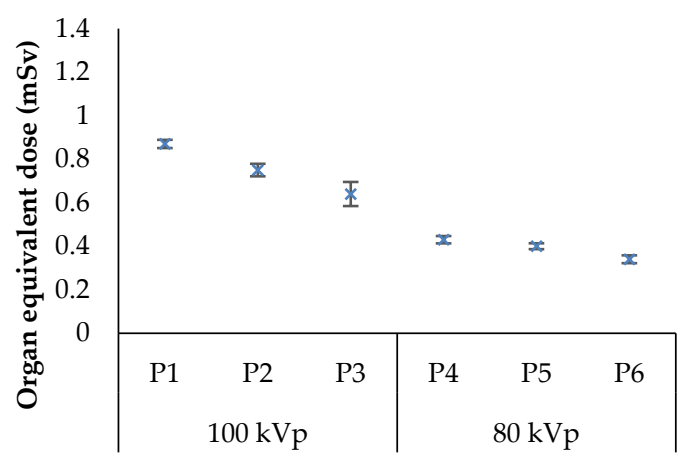

CT CAP Protocols (d)

Stomach

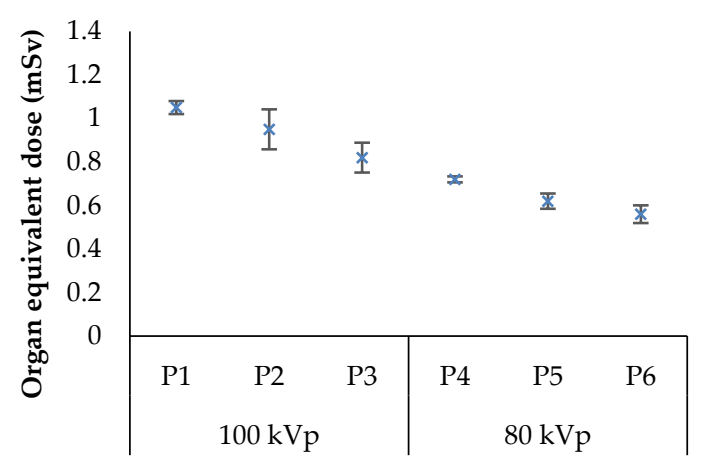

CT CAP Protocols

(f)

Skin

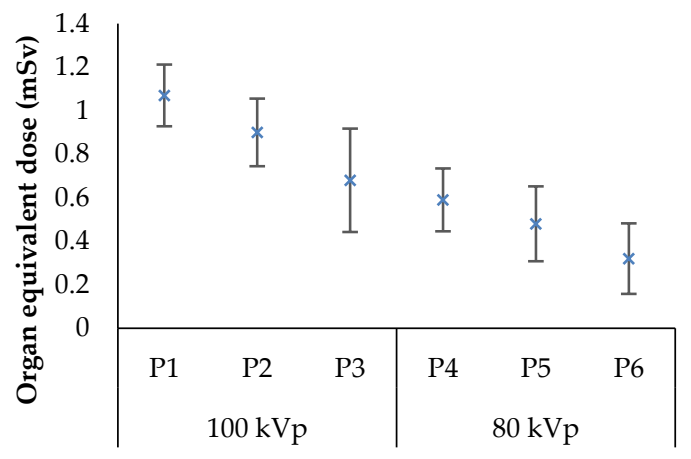

CT CAP Protocols

Figure 3. The mean organ-absorbed doses of standard pediatric CT CAP examinations with varying tube potential and tube current in: (a) Thyroid, (b) lungs, (c) liver, (d) stomach, (e) gonads, and (f) skin.

Table 3. Organ Equivalent Dose from TLD measurement from optimization protocols.

\begin{tabular}{ccccc}
\hline \multirow{2}{*}{ Selected Organs } & \multicolumn{4}{c}{ Organ Equivalent Dose $(\mathbf{m S v})$} \\
\cline { 2 - 5 } & $\mathbf{P 1 ( R )}$ & P7 $(\mathbf{S . C}=\mathbf{1 . 2})$ & $\mathbf{P 8}(\mathbf{P}=\mathbf{1 . 2})$ & P9 (Off TCM) \\
\hline Thyroid & $0.49 \pm 0.03$ & $0.36 \pm 0.36$ & $0.58 \pm 0.23$ & $4.25 \pm 0.27$ \\
Lungs & $0.88 \pm 0.12$ & $0.82 \pm 0.14$ & $0.91 \pm 0.13$ & $6.2 \pm 0.55$ \\
Liver & $1.22 \pm 0.13$ & $1.10 \pm 0.07$ & $1.30 \pm 0.04$ & $7.10 \pm 0.54$ \\
Stomach & $1.05 \pm 0.03$ & $0.97 \pm 0.07$ & $1.18 \pm 0.11$ & $6.91 \pm 0.42$ \\
Gonads & $0.87 \pm 0.02$ & $0.79 \pm 0.04$ & $0.90 \pm 0.02$ & $6.35 \pm 0.10$ \\
Skin & $1.07 \pm 0.14$ & $1.04 \pm 0.17$ & $1.15 \pm 0.11$ & $6.06 \pm 0.74$ \\
\hline $\mathrm{R}=$ Routine, S.C = Slice Collimation, P = Pitch, TCM = Tube Current Modulation.
\end{tabular}

Table 4. A comparison of all objective image quality parameter values acquired in different CT CAP protocols.

\begin{tabular}{|c|c|c|c|c|c|c|c|c|c|}
\hline \multirow{2}{*}{ Image Quality } & \multicolumn{9}{|c|}{ CT CAP Protocol } \\
\hline & P1 & P2 & P3 & P4 & P5 & P6 & P7 & P8 & P9 \\
\hline $\begin{array}{l}\text { CT Number } \\
\text { (Mean) }\end{array}$ & $22.5 \pm 0.9$ & $22.3 \pm 0.9$ & $19.9 \pm 1.9$ & $19.5 \pm 0.8$ & $20.3 \pm 1.9$ & $10.1 \pm 1.3$ & $22.6 \pm 0.5$ & $22.0 \pm 0.9$ & $21.4 \pm 0.9$ \\
\hline $\begin{array}{c}\text { Noise (SD) } \\
\text { SNR } \\
\text { CNR } \\
\text { FOM }\end{array}$ & $\begin{array}{c}8.9 \pm 1.1 \\
2.54 \pm 0.4 \\
0.71 \pm 0.1 \\
3.65\end{array}$ & $\begin{array}{c}9.3 \pm 0.9 \\
2.41 \pm 0.2 \\
0.69 \pm 0.1 \\
3.90\end{array}$ & $\begin{array}{c}10.5 \pm 1.6 \\
1.93 \pm 0.4 \\
0.47 \pm 0.1 \\
2.97\end{array}$ & $\begin{array}{c}10.8 \pm 2.3 \\
1.87 \pm 0.4^{*} \\
0.59 \pm 0.1 \\
3.59\end{array}$ & $\begin{array}{c}11.5 \pm 0.6 \\
1.77 \pm 0.1^{*} \\
0.45 \pm 0.1^{*} \\
3.54\end{array}$ & $\begin{array}{c}13.1 \pm 0.6 \\
0.77 \pm 0.1 * \\
0.61 \pm 0.1 \\
0.75\end{array}$ & $\begin{array}{c}7.4 \pm 1.0 \\
3.10 \pm 0.4 \\
0.54 \pm 0.1 \\
5.73\end{array}$ & $\begin{array}{c}8.8 \pm 0.7 \\
2.51 \pm 0.2 \\
0.23 \pm 0.1^{*} \\
3.73\end{array}$ & $\begin{array}{c}3.2 \pm 0.2 \\
6.66 \pm 0.5 \text { * } \\
1.34 \pm 0.2 \text { * } \\
\quad 6.47\end{array}$ \\
\hline $\begin{array}{l}\text { Dunnett } t \text {-tests } \\
(p \text { value })\end{array}$ & Control & 1.000 & 0.058 & 0.611 & 0.028 & 0.779 & 0.255 & 0.000 & 0.000 \\
\hline
\end{tabular}

${ }^{*} p$-value $<0.05$ 
(a)

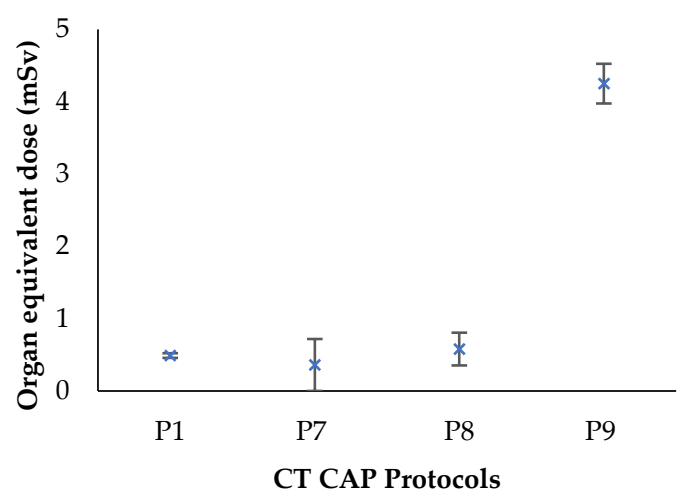

(c)

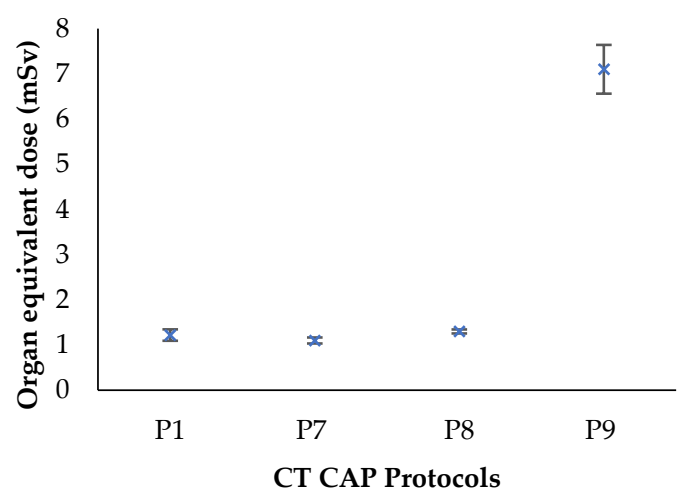

(e)

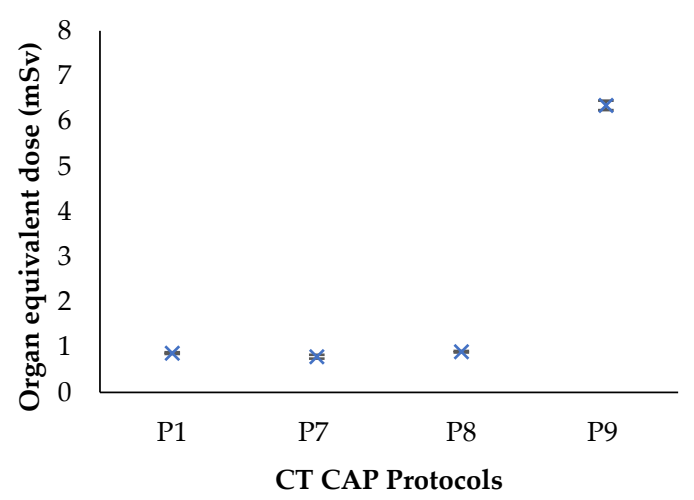

(b)

Lungs

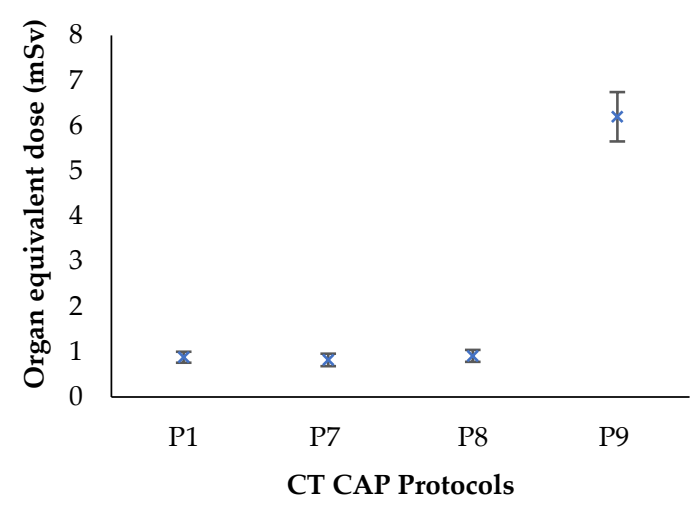

(d)

Stomach

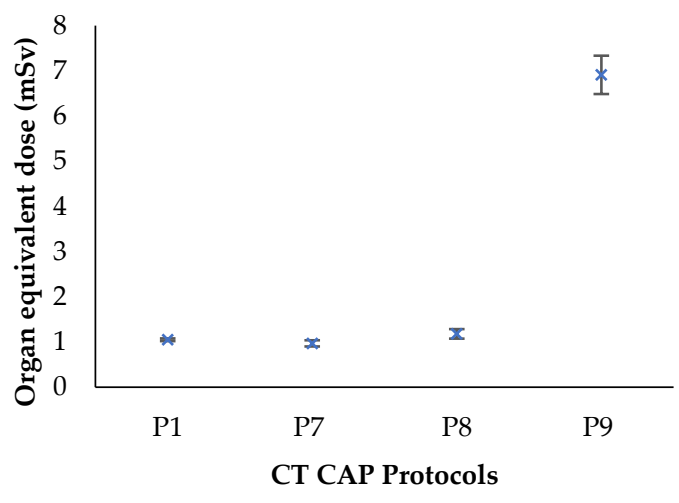

(f)

Skin

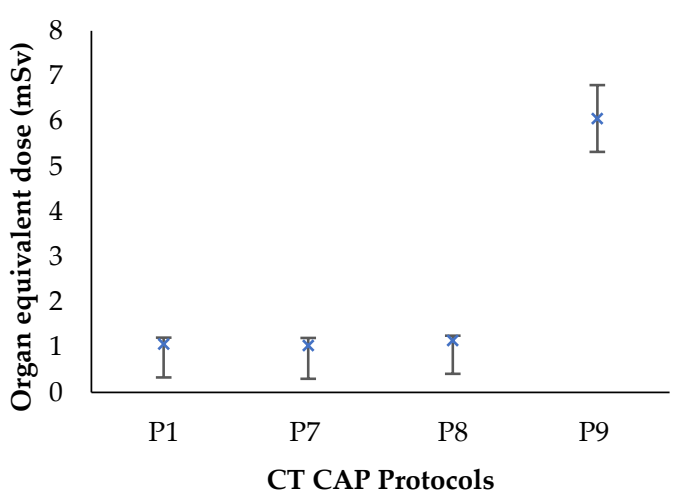

Figure 4. The mean organ-absorbed doses from the modification of standard pediatric CT CAP protocols in: (a) Thyroid, (b) lungs, (c) liver, (d) stomach, (e) gonads, and (f) skin.

\section{Discussion}

Our aim in this work is to correlate between dose and image quality metrics in pediatric while implementing CT optimization protocols. Among radiation dose metrics, organ equivalent dose is considered one of the best indicators for characterizing patient radiation burden $[10,32]$. Several researchers have been inspired to reduce the risk by altering the variable of scan parameters, including growing the pitch factor, increasing slice collimation, choosing tube voltage, and utilizing automatic tube current modulation (ATCM) feature [33-35]. 
In this work, we have measured organ-absorbed dose in pediatric patients by inserting a TL dosimeter into the hole designed at the slab of the one-year-old anthropomorphic phantom to represent specific radiosensitive organ parts. The CT CAP protocol was selected because of the high frequency performed amongst pediatric patients and the rise in repetitive scans due to the standard protocol used for cancer staging. Nevertheless, patients with early detection of malignancy can have multiple follow-up CT scans with elevated combined sensitivity to radiation, contributing to a possibility of radiation-related illness. Consequently, there is the concern as scan length that includes more than one part of the body may lead to the pediatric patient's over-exposure if CT parameter optimization has not been implemented.

The highest organ-absorbed dose was presented in the liver, skin, stomach, and lungs as the site of these organs was situated in the primary beam. As CT CAP is often applied as a routine $\mathrm{CT}$ examination in the pediatric population due to its benefit in revealing diseases in the lungs, liver, stomach, and deliberately for cancer staging, this can contribute to a rise in cancer incidence. The modification in CT parameter by lowering the tube voltage and tube current results in substantially different organ equivalent dose. Another research found that small adjustments in tube voltage might significantly reduce the radiation exposure and raise the quantum mottle resulting in increased image noise [36,37].

However, lowering the capacity of the X-ray tube is an acceptable operation to evaluate iodine structures as a consequence of the rise in iodine attenuation energy due to the proximity of the K-edge of iodine and the photoelectric influence. The optimum kilovoltage for a CT analysis should be selected depending on the imaging task and patient habitus [32]. Furthermore, the practice of low tube potential technique with a proper selection of $\mathrm{mAs}$ value will improve contrast resolution in a patient with a smaller body thickness. The decrease in tube voltage from 120 to $100 \mathrm{kVp}$ and $80 \mathrm{kVp}$ is the best range tube potential in pediatric CT chest, abdominal and pelvic examination without deteriorating CNR and SNR consistency index.

The alteration of $\mathrm{CT}$ scan parameter by reducing the pitch factor from 1.4 to 1.2 slightly increased the organ-absorbed dose without affecting image quality. This was supported by Lambert et al. [38], where the increased pitch factor on selected pediatric CT parameter may decrease the radiation output. As presented in P8, there is no significant difference $(p>0.05)$ in organ-absorbed dose albeit it was slightly increased compared to the routine protocol (P1) and also image diagnostic quality in terms of image noise and SNR value in this study. However, the CNR was found to be varied. Studies have shown that high-pitch CT is not only effective for cardiac imaging, but also for routine chest and abdominal CT analysis by minimizing radiation sensitivity while retaining diagnostic image consistency $[33,39]$. Moreover, the efficient high pitch value was found along with the iterative reconstruction algorithm for decreased radiation exposure and image noise, while tube current (mAs) stayed unchanged $[40,41]$.

Different size of the detector and slice collimation setting in P7 resulted in declining organ-absorbed dose in pediatric CT CAP analysis by contrasting the organ dose in all protocols. Besides dose reduction, it also indicates that there is no significant difference in image quality. According to the previous study reports, apart from growing pitch factor and reducing tube current and tube voltage in the optimization phase, increasing slice collimation is a technique to minimize radiation dose without sacrificing image quality performance [41,42]. Furthermore, previous research indicates that increased beam collimation resulted in a decrease of around $17 \%$ over-ranging in CT study, which may prevent overexposure to patients and decreased dose to radiosensitive organs beyond primary beam [43]. However, a wider detector z-coverage screening applied may increase radiation scattering. Scattered radiation can cause hypodense artefacts, affect the stability of the CT number, and the scatter-induced noise in the images may decrease the CNR [44].

The radiation dose metrics in terms of $\mathrm{CTDI}_{\mathrm{vol}}$, DLP, E and organ-absorbed dose results were substantially higher in P9. The body habitus of children populations varied in size and weight. In addition to the patient's size, the patient's anatomy has a major effect 
on X-ray attenuation and noise. At most children's health centres, CT CAP was applied for further investigation to determine the metastasis of cancer to the patients who were already diagnosed with primary cancer diseases. With the introduction of automatic tube current modulation (ATCM), this technique was used to optimize the radiation dose, particularly amongst pediatric patients. Moreover, with using TCM, $80 \%$ of organ-absorbed dose was reduced in pediatric CT CAP examination. This study was similar to the previous study shows by using TCM, $68 \%$ of effective dose reduce in CT CAP examination [40]. Therefore, the patient size, the shape, and anatomy of the patient has a significantly effect on TCM in terms of declining radiation dose without sacrificing image quality $[4,24]$.

The adjustments to these scanning parameters were investigated as to how these influences the patient dosage and the performance of CNR, SNR, and image noise. Nevertheless, there is a trade-off between image quality and dosage. The larger dose leading to the higher signal improves contrast resolution, lowering the noise and providing clearer depiction of low-contrast structures. Detection of low contrast information and lesions is restricted primarily by noise, which can be minimized by increasing the radiation exposure. Dose output of CT scanners operating in such modes can be described along with standard-dose indicators by image quality values. Hence, the trade-off between image quality and radiation dose were accessed by FOM based on SNR and CTDI $\mathrm{vol}_{\mathrm{vol}}$ in this study. Several studies explored the probability of dose reduction by optimization dependent on FOM approach $[14,45,46]$.

In addition, artificial intelligence has lately been commonly used to optimize radiationbased processes and has several benefits over conventional approaches. The usage of artificial intelligence contributes to refining photon radiation-based applications in both the medical and manufacturing industries [47-49]. Likewise, Machine Learning and Deep Learning a subset of artificial intelligence have been used in a number of applications to evaluate complicated data sets and to identify similarities and associations within those data without being directly configured [50,51].

This research has some limitations to list. First, this analysis just checked the alteration of the pediatric CT CAP protocols on a one-year-old anthropomorphic phantom. Therefore, the age variation in anthropomorphic phantom can be used to present radiation intensity and the dosage received by the organs of children owing to the variation of body size and habitus. Second, this research did not conduct a subjective evaluation of the accuracy of the picture by an expert radiologist. Third, the standard image reconstruction approach, which is Filter Back Projection (FBP) also known as Radon transform, was used for image processing since the $\mathrm{CT}$ scanner used in this study did not have an iterative reconstruction algorithm. The degree of image noise filtering is then reduced relative to the use of the iterative reconstruction process. The image quality by leveraging iterative reconstruction information is important to explain the impact on diagnostic quality subjectively as it has enhanced image quality, improve image clarity, and reduce image noise [52].

\section{Conclusions}

As a conclusion, the impact of the modification of acquisition parameter in pediatric $\mathrm{CT}$ CAP, indicates a potential reduction in a dose up to $30 \%$ to $80 \%$. Noting that, this study has focused on phantom study by selecting pertinent tube voltage, tube current, pitch value, slice collimation, and comparing ATCM technology. The attenuation-based treatment modifications are more effective than external patient features, such as patient age, weight, $\mathrm{BMI}$, or external measurements. The radiation-induced risk from current $\mathrm{CT}$ acquisition parameters is still relatively lower compared to natural background radiation. Even so, the justification for CT scan as well as optimization of the scanning parameters is still paramount for those patients who require multiple CT scans.

Author Contributions: Conceptualization, N.A.M. and M.K.A.K.; methodology, N.A.M.; software, M.K.A.K.; validation, Z.K., M.K.A.K. and J.H.D.W.; formal analysis, N.A.M. and H.A.H.; investigation, N.A.M.; resources, M.K.A.K.; data curation, Z.K. and H.A.H.; original draft preparation, N.A.M.; writing review and editing, M.K.A.K. and K.H.N.; visualization, M.K.A.K.; supervision, M.K.A.K.; 
project administration, Z.K.; funding acquisition, M.K.A.K. All authors have read and agreed to the published version of the manuscript.

Funding: This research was funded by Ministry of Higher Education (Malaysia) and Universiti Putra Malaysia with grant number FRGS/1/2020/STG07/UPM/02/3.

Institutional Review Board Statement: Not applicable.

Informed Consent Statement: Not applicable.

Acknowledgments: The authors are grateful to Mohd Kamil bin Mohd Fabell from UMMC for his technical assistance during this work.

Conflicts of Interest: The authors declare no conflict of interest.

\section{References}

1. Haleem, A.; Javaid, M. Role of CT and MRI in the design and development of orthopaedic model using additive manufacturing. J. Clin. Orthop. Trauma 2018, 9, 213-217. [CrossRef]

2. Wang, J.; Fleischmann, D. Improving spatial resolution at CT: Development, benefits, and pitfalls. Radiology 2018, $289,261-262$. [CrossRef] [PubMed]

3. Kubo, T. Vendor free basics of radiation dose reduction techniques for CT. Eur. J. Radiol. 2019, 110, 14-21. [CrossRef] [PubMed]

4. Laqmani, A.; Regier, M.; Veldhoen, S.; Backhaus, A.; Wassenberg, F.; Sehner, S.; Groth, M.; Nagel, H.-D.; Adam, G.; Henes, F.O. Improved image quality and low radiation dose with hybrid iterative reconstruction with $80 \mathrm{kV} \mathrm{CT}$ pulmonary angiography. Eur. J. Radiol. 2014, 83, 1962-1969. [CrossRef] [PubMed]

5. Papadakis, A.E.; Damilakis, J. Automatic tube current modulation and tube voltage selection in pediatric computed tomography: A phantom study on radiation dose and image quality. Invest. Radiol. 2019, 54, 265-272. [CrossRef]

6. Aw-Zoretic, J.; Seth, D.; Katzman, G.; Sammet, S. Estimation of effective dose and lifetime attributable risk from multiple head CT scans in ventriculoperitoneal shunted children. Eur. J. Radiol. 2014, 83, 1920-1924. [CrossRef] [PubMed]

7. Muhammad, N.; Karim, M.; Hassan, H.; Kamarudin, M.; Wong, J.; Ibahim, M. Estimation of effective dose and organ cancer risk from paediatric computed tomography thorax-Abdomen-Pelvis examinations. Radiat. Phys. Chem. 2019, 165, 108438. [CrossRef]

8. Al-Rammah, T.Y. CT radiation dose awareness among paediatricians. Ital. J. Pediatr. 2016, 42, 1-6. [CrossRef]

9. Krille, L.; Zeeb, H.; Jahnen, A.; Mildenberger, P.; Seidenbusch, M.; Schneider, K.; Weisser, G.; Hammer, G.; Scholz, P.; Blettner, M. Computed tomographies and cancer risk in children: A literature overview of CT practices, risk estimations and an epidemiologic cohort study proposal. Radiat. Environ. Biophys. 2012, 51, 103-111. [CrossRef]

10. Perisinakis, K.; Tzedakis, A.; Pouli, S.; Spanakis, K.; Hatzidakis, A.; Damilakis, J. Comparison of patient dose from routine multiphase and dynamic liver perfusion CT studies taking into account the effect of iodinated contrast administration. Eur. J. Radiol. 2019, 110, 39-44. [CrossRef] [PubMed]

11. Applegate, K.E.; Frush, D.P. Image gently: A Decade of international collaborations to promote appropriate imaging for children. J. Am. Coll. Radiol. 2017, 14, 956-957. [CrossRef]

12. Parakh, A.; Kortesniemi, M.; Schindera, S.T. CT radiation dose management. Radiology 2016, 280, 663-673. [CrossRef]

13. Alqahtani, S.J.M.; Welbourn, R.; Meakin, J.R.; Palfrey, R.M.; Rimes, S.J.; Thomson, K.; Knapp, K.M. Increased radiation dose and projected radiation-related lifetime cancer risk in patients with obesity due to projection radiography. J. Radiol. Prot. 2019, 39, 38-53. [CrossRef] [PubMed]

14. Racine, D.; Ryckx, N.; Ba, A.; Becce, F.; Viry, A.; Verdun, F.R.; Schmidt, S. Task-based quantification of image quality using a model observer in abdominal CT: A multicentre study. Eur. Radiol. 2018, 28, 5203-5210. [CrossRef]

15. Hashim, S.; Karim, M.K.A.; Bakar, K.; Sabarudin, A.; Chin, A.; Saripan, M.; Bradley, D. Evaluation of organ doses and specific k effective dose of 64-slice CT thorax examination using an adult anthropomorphic phantom. Radiat. Phys. Chem. 2016, 126, 14-20. [CrossRef]

16. Gao, Y.; Quinn, B.; Mahmood, U.; Long, D.; Erdi, Y.; St. Germain, J.; Pandit-Taskar, N.; Xu, G.; Bolch, W.; Dauer, L. A comparison of pediatric and adult CT organ dose estimation methods. BMC Med. Imaging 2017, 17, 28. [CrossRef]

17. Goodman, T.R.; Mustafa, A.; Rowe, E. Pediatric CT radiation exposure: Where we were, and where we are now. Pediatr. Radiol. 2019, 49, 469-478. [CrossRef]

18. Stamm, G.; Nagel, H.D. CT-expo-A novel program for dose evaluation in CT. RöFo 2002, 174, 1570-1576. (In German)

19. Hoang, J.K.; Reiman, R.E.; Nguyen, G.B.; Januzis, N.; Chin, B.B.; Lowry, C.; Yoshizumi, T.T. Lifetime attributable risk of cancer from radiation exposure during parathyroid imaging: Comparison of 4D CT and parathyroid scintigraphy. Am. J. Roentgenol. 2015, 204, W579-W585. [CrossRef] [PubMed]

20. Samei, E.; Tian, X.; Segars, W.P.; Frush, D.P. Radiation risk index for pediatric CT: A patient-derived metric. Pediatr. Radiol. 2017, 47, 1737-1744. [CrossRef] [PubMed]

21. Park, C.; Gruber-Rouh, T.; Leithner, D.; Zierden, A.; Albrecht, M.H.; Wichmann, J.L.; Bodelle, B.; Elsabaie, M.; Scholtz, J.-E.; Kaup, M.; et al. Single-source chest-abdomen-pelvis cancer staging on a third generation dual-source CT system: Comparison of automated tube potential selection to second generation dual-source CT. Cancer Imaging 2016, 16, 33. [CrossRef] [PubMed] 
22. Gao, Y.; Quinn, B.; Pandit-Taskar, N.; Behr, G.; Mahmood, U.; Long, D.; Xu, G.; St. Germain, J.; Dauer, L. Patient-specific organ and effective dose estimates in pediatric oncology computed tomography. EJMP 2018, 45, 146-155. [CrossRef]

23. Abdulkadir, M.K.; Mat Rahim, N.A.Y.; Mazlan, N.S.; Daud, N.M.; Shuaib, I.L.; Osman, N.D. Dose optimisation in paediatric CT examination: Assessment on current scanning protocols associated with radiation dose. Radiat. Phys. Chem. 2020, 171, 108740. [CrossRef]

24. Halid, B.; Karim, M.K.A.; Sabarudin, A.; Bakar, K.A.; Shariff, N.D. Assessment of lifetime attributable risk of stomach and colon cancer during abdominal ct examinations based on Monte Carlo simulation. In Proceedings of the 8th European Medical and Biological Engineering Conference; Springer International Publishing: New York, NY, USA, 2017; pp. 455-459.

25. Seeram, E.; Davidson, R.; Bushong, S.; Swan, H. Optimizing the exposure indicator as a dose management strategy in computed radiography. Radiol. Technol. 2016, 87, 380-391.

26. Tonkopi, E.; Duffy, S.; Abdolell, M.; Manos, D. Diagnostic reference levels and monitoring practice can help reduce patient dose from ct examinations. Am. J. Roentgenol. 2017, 208, 1073-1081. [CrossRef] [PubMed]

27. Karim, M.K.A.; Hashim, S.; Bradley, D.A.; Bahruddin, N.A.; Ang, W.C.; Salehhon, N. Assessment of knowledge and awareness among radiology personnel regarding current computed tomography technology and radiation dose. J. Phys. Conf. Ser. 2016, 694, 012031. [CrossRef]

28. Isa, I.; Rahmat, S.; Dom, S.; Kayun, Z.; Karim, M. The effects of mis-centering on radiation dose during CT head examination: A phantom study. J. X-ray Sci. Technol. 2019, 27, 631-639. [CrossRef]

29. Shaffiq Said Rahmat, S.M.; Abdul Karim, M.K.; Che Isa, I.N.; Abd Rahman, M.A.; Noor, N.M.; Hoong, N.K. Effect of miscentering and low-dose protocols on contrast resolution in computed tomography head examination. Comput. Biol. Med. 2020, 123, 103840. [CrossRef]

30. Harun, H.; Karim, M.; Abbas, Z.; Sabarudin, A.; Muniandy, S.; Razak, H.; Ng, K. The influence of iterative reconstruction level on image quality and radiation dose in CT pulmonary angiography examinations. Radiat. Phys. Chem. 2021, 178, 108989. [CrossRef]

31. Harun, H.H.; Karim, M.K.A.; Abbas, Z.; Rahman, M.A.A.; Sabarudin, A.; Ng, K.H. Association of radiation doses and cancer risks from ct pulmonary angiography examinations in relation to body diameter. Diagnostics 2020, 10, 681. [CrossRef] [PubMed]

32. McCollough, C.; Leng, S. Use of artificial intelligence in computed tomography dose optimisation. Ann. ICRP 2020, 49, 113-125. [CrossRef]

33. Barrera, C.A.; Otero, H.J.; White, A.M.; Saul, D.; Biko, D.M. Image quality and radiation dose of ECG-triggered High-Pitch Dual-Source cardiac computed tomography angiography in children for the evaluation of central vascular stents. Int. J. Cardiovasc. Imaging 2019, 35, 367-374. [CrossRef]

34. Cardenas, C.E.; McCarroll, R.E.; Court, L.E.; Elgohari, B.A.; Elhalawani, H.; Fuller, C.D.; Kamal, M.J.; Meheissen, M.A.M.; Mohamed, A.S.R.; Rao, A.; et al. Deep learning algorithm for auto-delineation of high-risk oropharyngeal clinical target volumes with built-in dice similarity coefficient parameter optimization function. Int. J. Radiat. Oncol. Biol. Phys. 2018, 101, 468-478. [CrossRef]

35. Gomez-Cardona, D.; Hayes, J.W.; Zhang, R.; Li, K.; Cruz-Bastida, J.P.; Chen, G.-H. Low-dose cone-beam CT via raw counts domain low-signal correction schemes: Performance assessment and task-based parameter optimization (Part II. Task-based parameter optimization). Med. Phys. 2018, 45, 1957-1969. [CrossRef] [PubMed]

36. Berlin, S.C.; Weinert, D.M.; Vasavada, P.S.; Martinez-Rios, C.; Parikh, R.A.; Wien, M.A.; Jordan, D.W.; Novak, R.D. Successful dose reduction using reduced tube voltage with hybrid iterative reconstruction in pediatric abdominal CT. Am. J. Roentgenol. 2015, 205, 392-399. [CrossRef] [PubMed]

37. Nagayama, Y.; Oda, S.; Nakaura, T.; Tsuji, A.; Urata, J.; Furusawa, M.; Utsunomiya, D.; Funama, Y.; Kidoh, M.; Yamashita, Y. Radiation dose reduction at pediatric CT: Use of low tube voltage and iterative reconstruction. Radiographics 2018, 38, 1421-1440. [CrossRef] [PubMed]

38. Lambert, J.W.; Phelps, A.S.; Courtier, J.L.; Gould, R.G.; MacKenzie, J.D. Image quality and dose optimisation for infant CT using a paediatric phantom. Eur. Radiol. 2015, 26, 1387-1395. [CrossRef]

39. Trattner, S.; Chelliah, A.; Prinsen, P.; Ruzal-Shapiro, C.B.; Xu, Y.; Jambawalikar, S.; Amurao, M.; Einstein, A.J. Estimating effective dose of radiation from pediatric cardiac CT angiography using a 64-MDCT scanner: New conversion factors relating dose-length product to effective dose. Am. J. Roentgenol. 2017, 208, 585-594. [CrossRef]

40. Gariani, J.; Martin, S.P.; Botsikas, D.; Becker, C.D.; Montet, X. Evaluating the effect of increased pitch, iterative reconstruction and dual source CT on dose reduction and image quality. Br. J. Radiol. 2018, 91. [CrossRef] [PubMed]

41. Booij, R.; Dijkshoorn, M.L.; Van Straten, M. Efficacy of a dynamic collimator for overranging dose reduction in a second- and third-generation dual source CT scanner. Eur. Radiol. 2017, 27, 3618-3624. [CrossRef]

42. Messerli, M.; Dewes, P.; Scholtz, J.-E.; Arendt, C.; Wildermuth, S.; Vogl, T.J.; Bauer, R.W. Evaluation of an adaptive detector collimation for prospectively ECG-triggered coronary CT angiography with third-generation dual-source CT. Eur. Radiol. 2018, 28, 2143-2150. [CrossRef] [PubMed]

43. Urikura, A.; Hara, T.; Yoshida, T.; Nishimaru, E.; Hoshino, T.; Nakaya, Y.; Endo, M. Overranging and overbeaming measurement in area detector computed tomography: A method for simultaneous measurement in volume helical acquisition. J. Appl. Clin. Med. Phys. 2019, 20, 160-165. [CrossRef]

44. Flohr, T. CT Systems. Curr. Radiol. Rep. 2013, 1, 52-63. [CrossRef] 
45. Chang, K.-P.; Hsu, T.-K.; Lin, W.-T.; Hsu, W.-L. Optimization of dose and image quality in adult and pediatric computed tomography scans. Radiat. Phys. Chem. 2017, 140, 260-265. [CrossRef]

46. Harun, H.H.; Karim, M.K.A.; Muhammad, N.A.; Razak, H.R.A.; Sabarudin, A.; Muniandy, S.C. Effect of iterative reconstruction algorithm associated with low contrast detectability performance from CT pulmonary angiography examinations. J. Phys. Conf. Ser. 2020, 012029. [CrossRef]

47. Meineke, A.; Rubbert, C.; Sawicki, L.M.; Thomas, C.; Klosterkemper, Y.; Appel, E.; Caspers, J.; Bethge, O.T.; Kröpil, P.; Antoch, G.; et al. Potential of a machine-learning model for dose optimization in CT quality assurance. Eur. Radiol. 2019, 29, 3705-3713. [CrossRef] [PubMed]

48. Roshani, M.; Phan, G.; Faraj, R.H.; Phan, N.-H.; Roshani, G.H.; Nazemi, B.; Corniani, E.; Nazemi, E. Proposing a gamma radiation based intelligent system for simultaneous analyzing and detecting type and amount of petroleum by-products. Nucl. Eng. Technol. 2020. [CrossRef]

49. Roshani, M.; Phan, G.; Roshani, G.H.; Hanus, R.; Nazemi, B.; Corniani, E.; Nazemi, E. Combination of X-ray tube and GMDH neural network as a nondestructive and potential technique for measuring characteristics of gas-oil-water three phase flows. Measurement 2021, 168, 108427. [CrossRef]

50. Alla Takam, C.; Samba, O.; Tchagna Kouanou, A.; Tchiotsop, D. Spark architecture for deep learning-based dose optimization in medical imaging. Inform. Med. Unlocked. 2020, 19, 100335. [CrossRef]

51. Singh, R.; Wu, W.; Wang, G.; Kalra, M.K. Artificial intelligence in image reconstruction: The change is here. Phys. Med. 2020, 79, 113-125. [CrossRef]

52. Verdun, F.R.; Racine, R.D.; Ott, J.G.; Tapiovaara, T.M.; Toroi, P.; Bochud, F.O.; Veldkamp, W.J.H.; Schegerer, A.A.; Bouwman, R.W.; Giron, I.H.; et al. Image quality in CT: From physical measurements to model observers. Phys. Med. 2015, 31, 823-843. [CrossRef] [PubMed] 